\title{
Amorphous Alloy Architectures in Pore Walls: Mesoporous Amorphous NiCoB Alloy Spheres with Controlled Compositions via a Chemical Reduction
}

Yunqing Kang, Bo Jiang,* Juanjuan Yang, Zhe Wan, Jongbeom Na, Qian Li, Hexing Li, Joel Henzie, Yoshio Sakka, Yusuke Yamauchi* and Toru Asahi*

Yunqing Kang, Toru Asahi*

Faculty of Science and Engineering, Waseda University, 3-4-1 Okubo, Shinjuku, Tokyo 169-8555, Japan

Yunqing Kang, Qian Li, Joel Henzie, Yusuke Yamauchi*

JST-ERATO Yamauchi Materials Space-Tectonics Project and International Center for Materials Nanoarchitectonics (WPI-MANA), National Institute for Materials Science (NIMS), 1-1 Namiki, Tsukuba, Ibaraki 305-0044, Japan

Bo Jiang*, Juanjuan Yang, Zhe Wan, Hexing Li

The Education Ministry Key Lab of Resource Chemistry and Shanghai Key Laboratory of Rare Earth Functional Materials, Shanghai Normal University, Shanghai 200234, China

Jongbeom Na, Yusuke Yamauchi*

School of Chemical Engineering and Australian Institute for Bioengineering and Nanotechnology (AIBN), The University of Queensland, Brisbane, QLD 4072, Australia

Qian Li

School of Chemistry and Chemical Engineering, Shanghai Key Laboratory of Electrical Insulation and Thermal Ageing, Shanghai Jiao Tong University, 800 Dongchuan Road, Shanghai, 200240 China.

Yoshio Sakka

Research Center for Functional Materials, National Institute for Materials Science (NIMS), 1-2-1 Sengen, Tsukuba, Ibaraki 305-0047, Japan

Yusuke Yamauchi*, Toru Asahi*

JST-ERATO Yamauchi Materials Space-Tectonics Project, Kagami Memorial Research Institute for Materials Science and Technology, Waseda University, 2-8-26 Nishiwaseda, Shinjuku-ku, Tokyo 169-0051, Japan 


\section{CONTENTS}

Figure S1. Photo showing the Tyndall effect in solutions with different compositions

Figure S2. SEM images, particle/pore size distribution, and thickness of the pore wall of meso$\mathrm{Ni}_{10.0} \mathrm{Co}_{74.5} \mathrm{~B}_{15.5} \mathrm{AASs}$.

Figure S3. SEM images of non-porous $\mathrm{Ni}_{10.0} \mathrm{Co}_{74.5} \mathrm{~B}_{15.5}$.

Figure S4. SEM images and sphere/pore size distribution of the as-prepared samples with different compositions.

Figure S5. Photos of the reaction process with different Ni ratios of meso-NiCoB AASs.

Figure S6. SEM of meso-NiCoB with high Ni content.

Figure S7. The XPS of Co $2 p 3 / 2$ of the meso-CoB sample.

Figure S8. The photos of meso-Ni ${ }_{10.0} \mathrm{Co}_{74.5} \mathrm{~B}_{15.5} \mathrm{AASs}$ prepared only using DMAB

Figure S9. The structural formula of $\mathrm{Bu}_{4} \mathrm{PBr}$.

Figure S10. The SEM pictures of meso- $\mathrm{Ni}_{10.0} \mathrm{Co}_{74.5} \mathrm{~B}_{15.5} \mathrm{AASs}$ prepared with different amounts of $\mathrm{Bu}_{4} \mathrm{PBr}$.

Figure S11. The photos of $0.3 \mathrm{~g} \mathrm{Bu} 4 \mathrm{PBr}$ dissolve in a mixed solution of $\mathrm{Co}(\mathrm{AC})_{2}$ and $\mathrm{Ni}(\mathrm{AC})_{2}$.

Figure S12. Photos of the reduction process of meso- $\mathrm{Ni}_{10.0} \mathrm{Co}_{74.5} \mathrm{~B}_{15.5}$ with and without $\mathrm{Bu} 4 \mathrm{PBr}$.

Figure S13. SEM images of meso- $\mathrm{Ni}_{10.0} \mathrm{Co}_{74.5} \mathrm{~B}_{15.5} \mathrm{AASs}$ prepared replacing $\mathrm{Bu}_{4} \mathrm{PBr}$ with $\mathrm{Bu}_{4} \mathrm{PCl}$ and $\mathrm{Bu}_{4} \mathrm{NBr}$.

Figure S14. The magnetic recyclability of the meso-Ni ${ }_{10.0} \mathrm{Co}_{74.5} \mathrm{~B}_{15.5} \mathrm{AASs}$.

Figure S15. The XRD and SEM image of meso-Ni ${ }_{10.0} \mathrm{CO}_{74.5} \mathrm{~B}_{15.5} \mathrm{AAs}$ after the stability test.

Table S1. The relationship between the final elemental concentration and the precursors.

Table S2. Catalytic activities from the hydrolysis of $A B$ catalyzed by as-prepared samples with different metal ratios.

Table S3. The hydrolysis of $\mathrm{AB}$ performance of the as-prepared $\mathrm{Ni}_{10.0} \mathrm{Co}_{74.5} \mathrm{~B}_{15.5}$ prepared with different structure-directing agents.

Table S4. The catalytic activity of different Co-based catalysts used for the hydrolytic dehydrogenation of $\mathrm{AB}$.

\section{References}



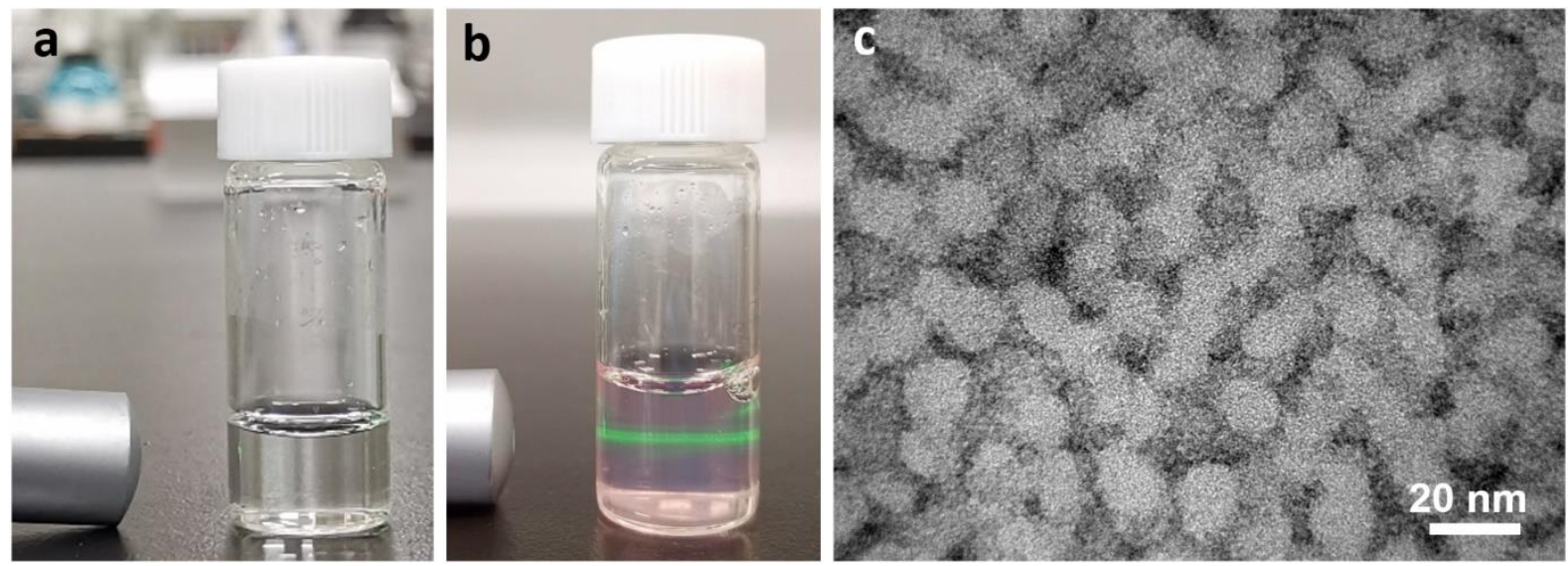

Figure S1. Photo showing the Tyndall effect in solutions with different compositions: (a) PS- $b$-PEO dissolved in DMF, (b) PS- $b$-PEO dissolved in $\mathrm{DMF}+\mathrm{Ni}(\mathrm{AC})_{2}+\mathrm{Co}(\mathrm{AC})_{2}+\mathrm{Bu} 4 \mathrm{PBr}+\mathrm{DMAB}$. (c) TEM image of the polymeric micelles obtained from mixture solution of DMF + Water + PS- $b-\mathrm{PEO}$.
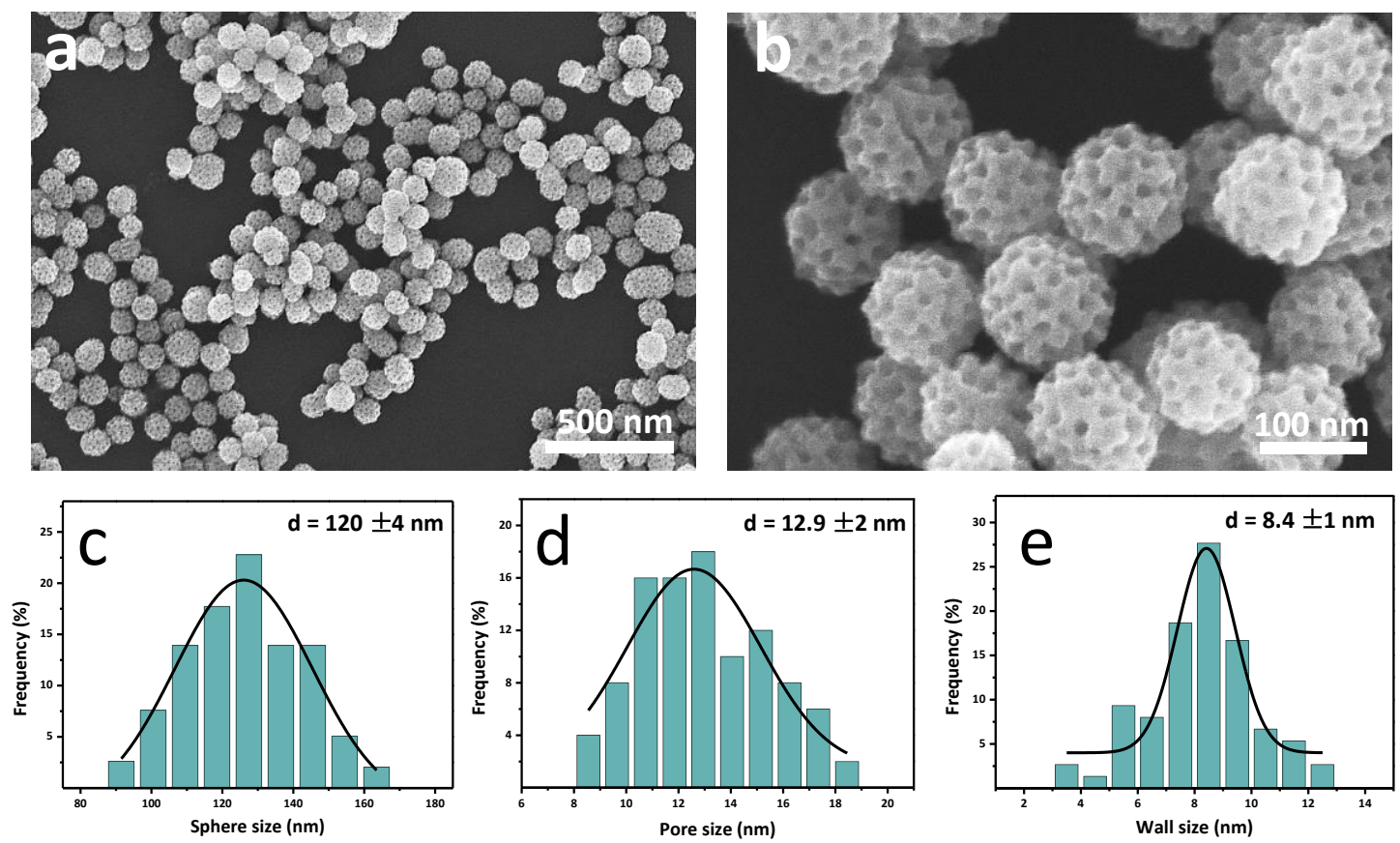

Figure S2. (a,b) SEM image, (c) particle size distribution, (d) pore size distribution, and (e) thickness of the pore wall of the typical meso- $\mathrm{Ni}_{10.0} \mathrm{Co}_{74.5} \mathrm{~B}_{15.5} \mathrm{AASs}$ sample. 

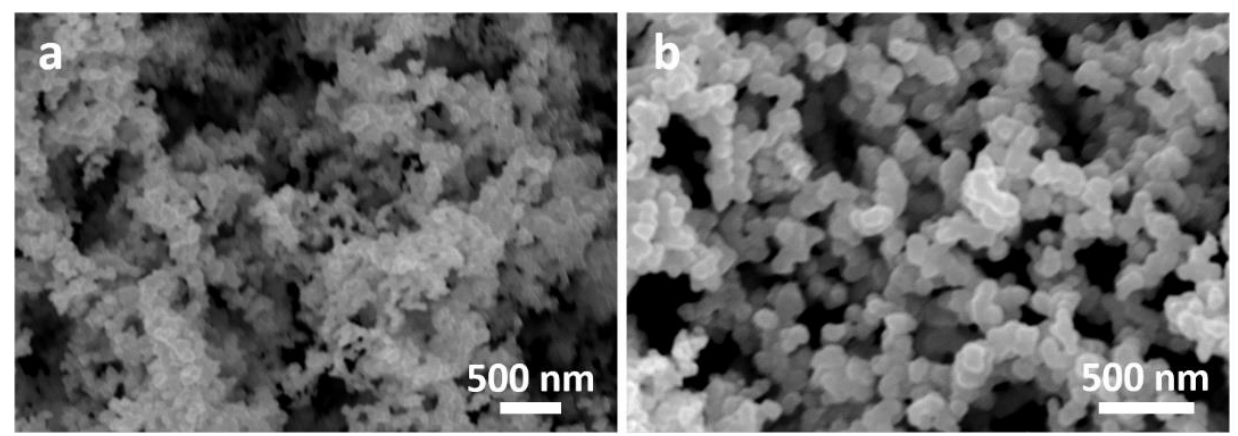

Figure S3. SEM images of (a) non-porous $\mathrm{Ni}_{10.0} \mathrm{Co}_{74.5} \mathrm{~B}_{15.5}-1$ and (b) non-porous $\mathrm{Ni}_{10.0} \mathrm{Co}_{74.5} \mathrm{~B}_{15.5}-2$ AAs prepared without using PS- $b$-PEO $+\mathrm{Bu}_{4} \mathrm{PBr}$ and $\mathrm{PS}-b$-PEO, respectively. 


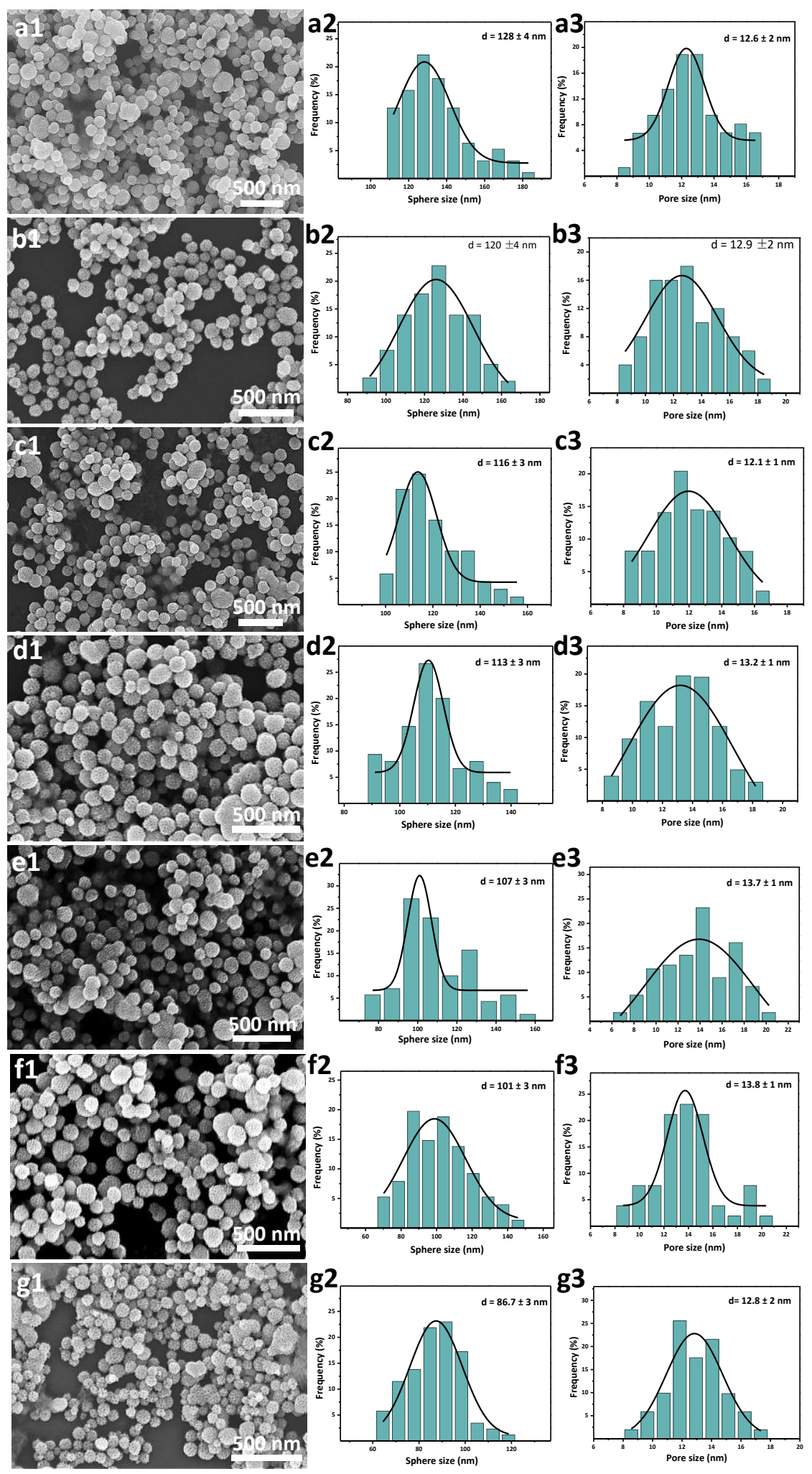

Figure S4. The SEM images (a1-g1), sphere size distribution (a2-g2), and pore size distribution (a3g3) of the as-prepared samples: (a) meso-Co ${ }_{85.0} \mathrm{~B}_{15.0}$, (b) meso-Ni $\mathrm{N}_{10.0} \mathrm{Co}_{74.5} \mathrm{~B}_{15.5}$, (c) meso$\mathrm{Ni}_{16.3} \mathrm{Co}_{67.8} \mathrm{~B}_{15.9}$, (d) meso-Ni24.0 $\mathrm{Co}_{60.00} \mathrm{~B}_{16.0}$, (e) meso-Ni40.0 $\mathrm{Co}_{41.7} \mathrm{~B}_{18.3}$, (f) meso-Ni63.1 $\mathrm{Co}_{16.1} \mathrm{~B}_{20.8}$ and (g) meso-Ni79.0 $\mathrm{B}_{21.0}$ AASs. 


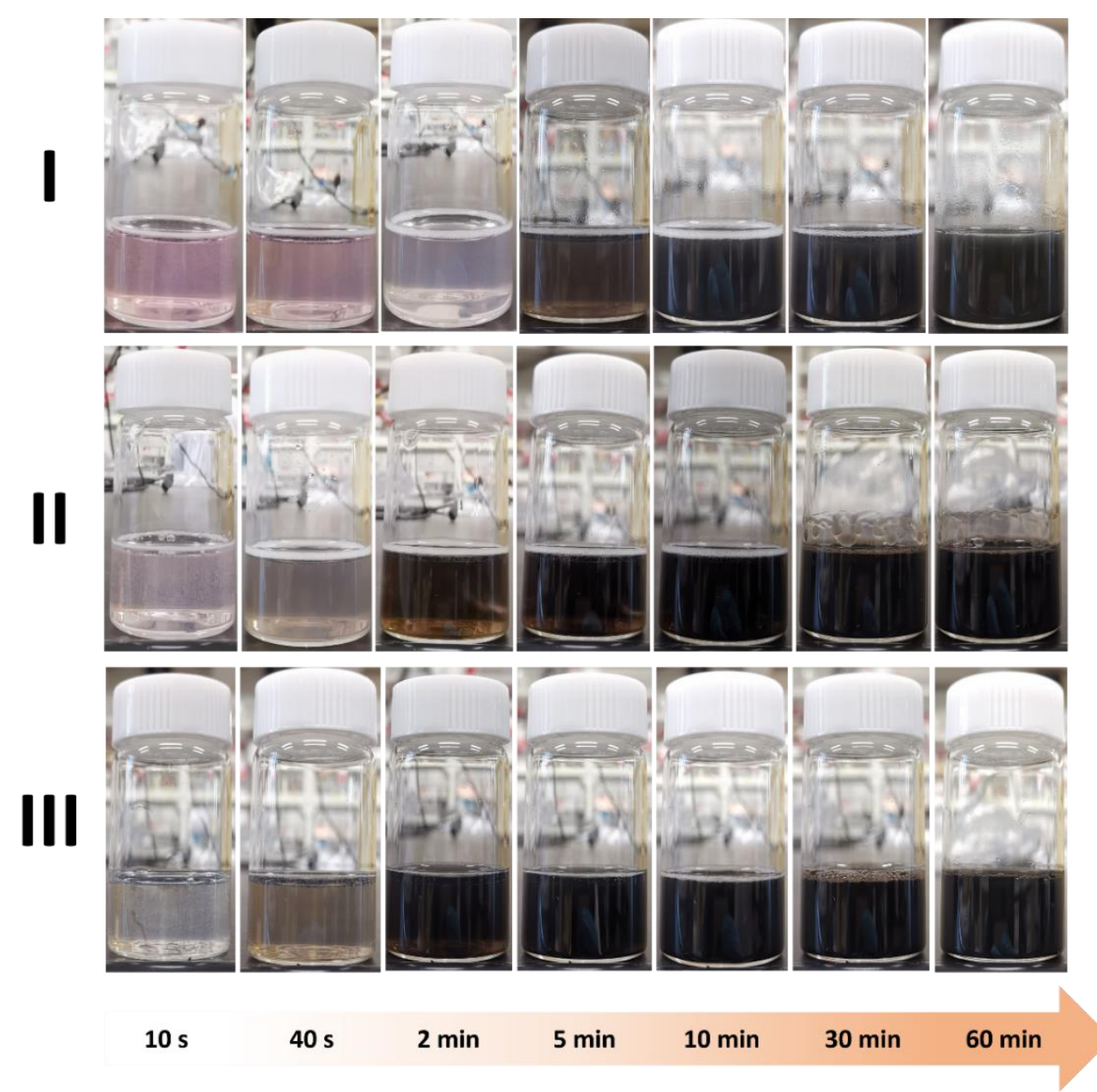

Figure S5. Photos of the reaction process with different Ni ratios of meso-NiCoB AASs. I) meso$\mathrm{Ni}_{10.0} \mathrm{Co}_{74.5} \mathrm{~B}_{15.5}$, II) meso-Ni40.0 $\mathrm{Co}_{41.7} \mathrm{~B}_{18.3}$, III) meso-Ni63.1 $\mathrm{Co}_{16.1} \mathrm{~B}_{20.8}$.

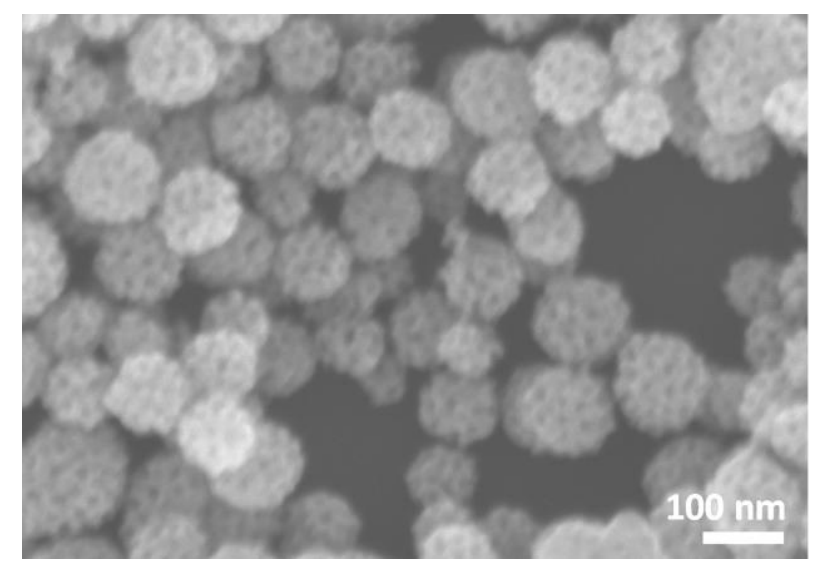

Figure S6. SEM of meso-NiCoB with high $\mathrm{Ni}$ content ( 90 at. $\%)$ prepared with precursors of $\mathrm{Ni}^{2+}$ : $\mathrm{Co}^{2+}=90: 10$ at the same preparation. 


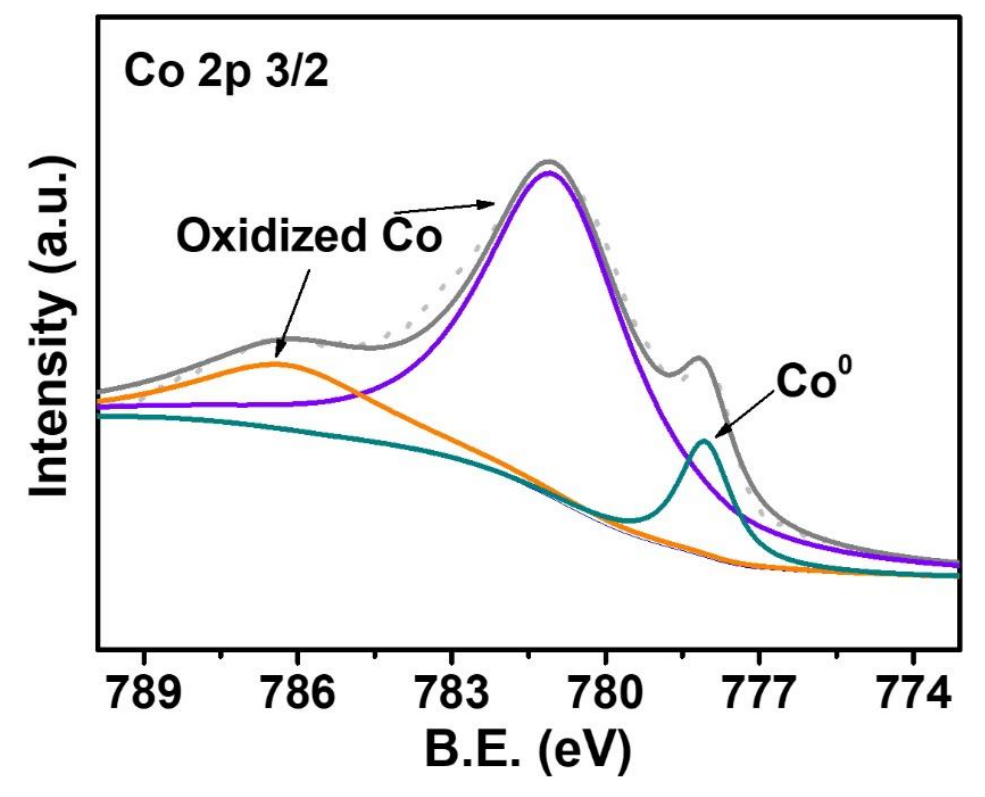

Figure S7. The XPS of Co $2 \mathrm{p} 3 / 2$ of the meso-CoB sample. (The area ratio of $\mathrm{Co}^{0} / \mathrm{Co}^{2+}$ is 2.68 and 0.52 for meso- $\mathrm{Co}_{85.0} \mathrm{~B}_{15.0}$ and meso- $\mathrm{Ni}_{10.0} \mathrm{Co}_{74.5} \mathrm{~B}_{15.5}$, respectively. The result shows pure meso$\mathrm{Co} 85.0 \mathrm{~B}_{15.0}$ surfaces are more occupied by oxides compared to meso-Ni ${ }_{10.0} \mathrm{Co}_{74.5} \mathrm{~B}_{15.5}$ alloy.)

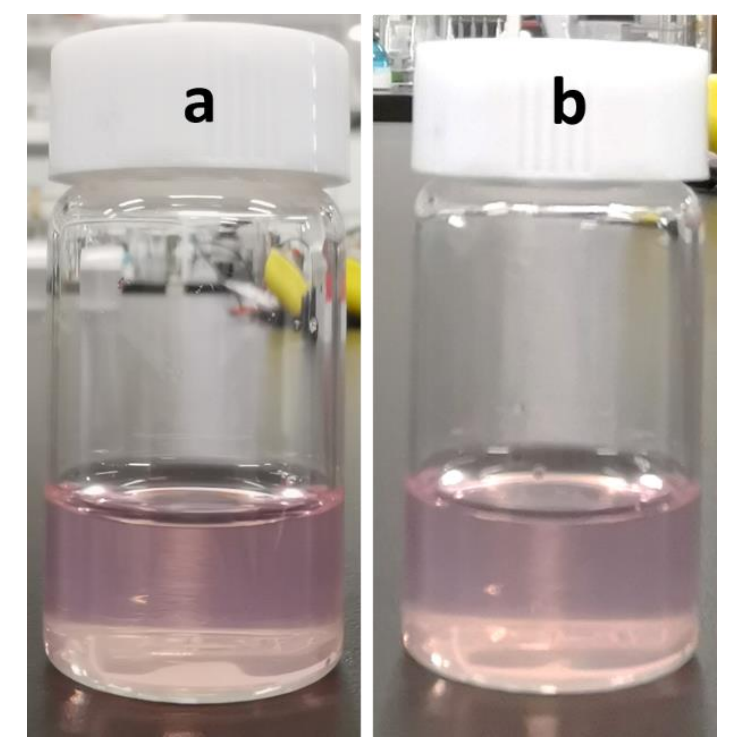

Figure S8. The photos of reaction solution prepared only using DMAB: (a) initial, (b) after $6.0 \mathrm{~h}$. 


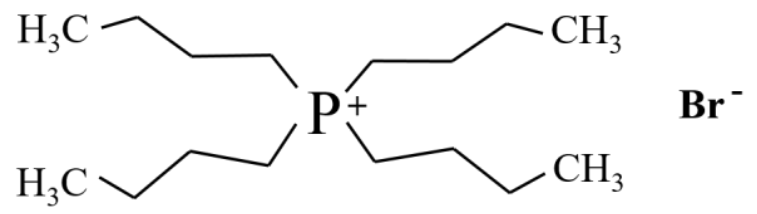

Figure S9. The structural formula of $\mathrm{Bu}_{4} \mathrm{PBr}$.
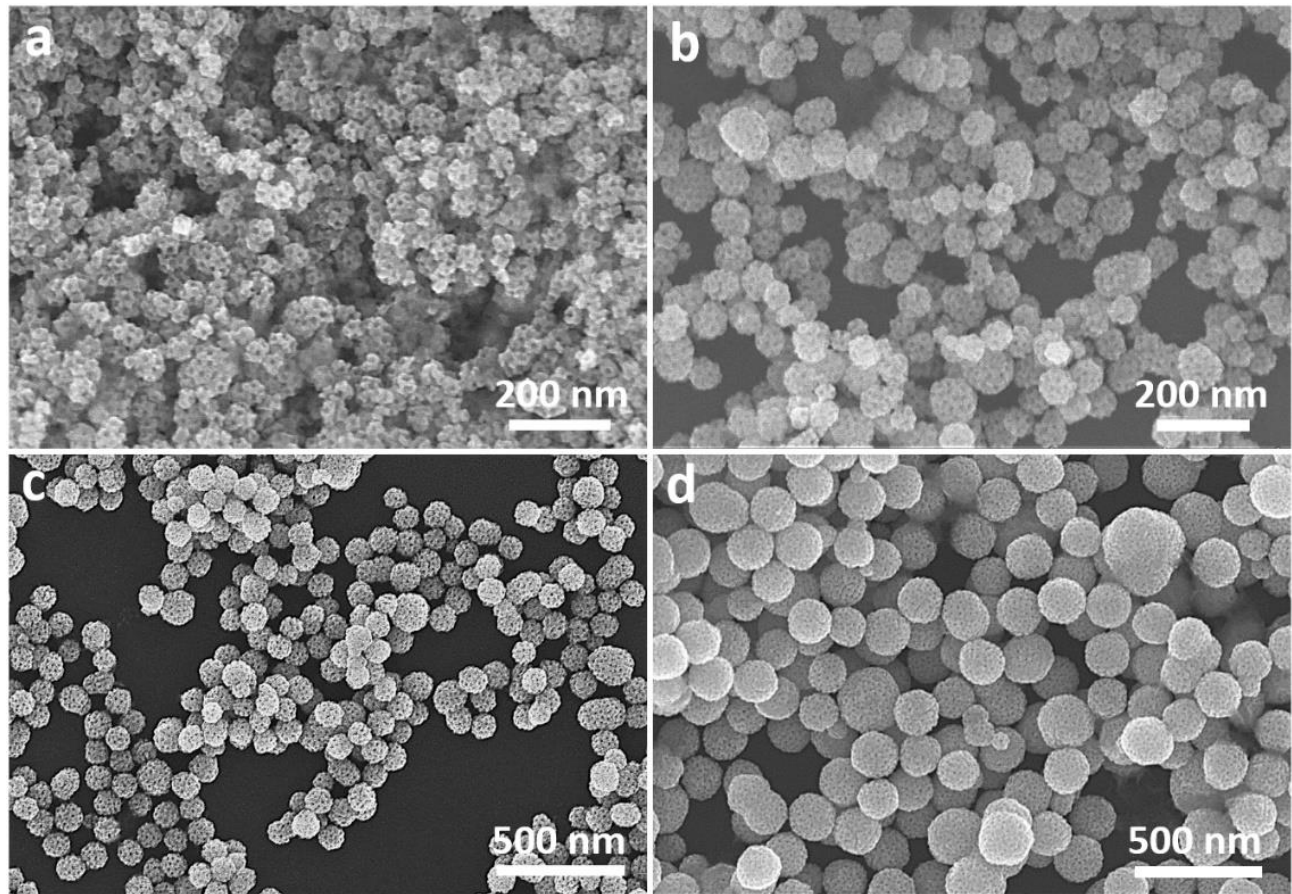

Figure S10. The SEM pictures of meso- $\mathrm{Ni}_{10.0} \mathrm{C}_{74.5} \mathrm{~B}_{15.5} \mathrm{AASs}$ prepared with different amounts of $\mathrm{Bu}_{4} \mathrm{PBr}$ of (a) $0 \mathrm{~g}$, (b) $0.2 \mathrm{~g}$, (c) $0.3 \mathrm{~g}$ and (d) $0.4 \mathrm{~g}$. 


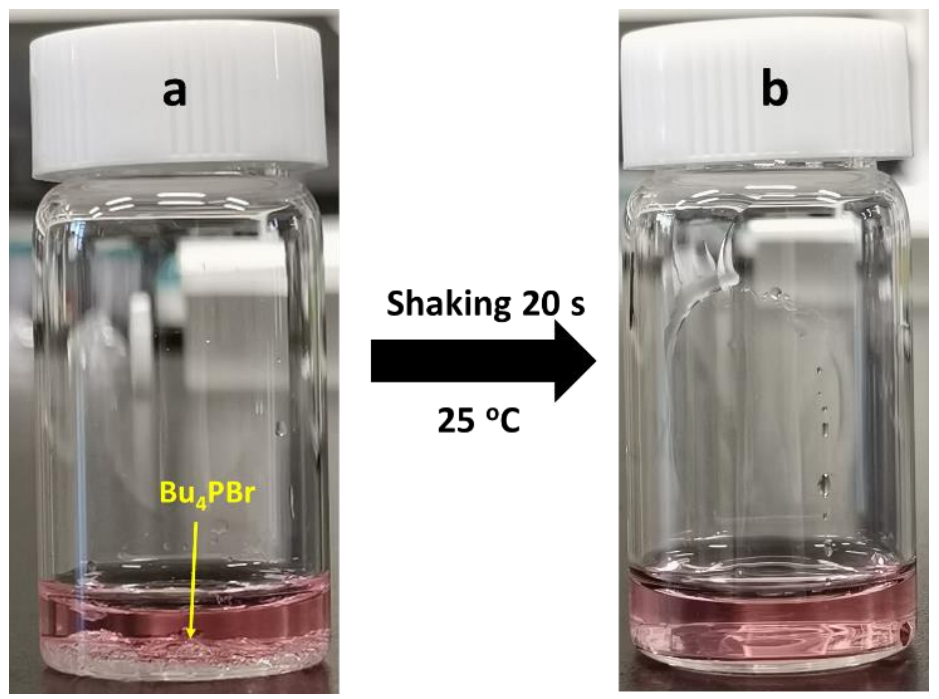

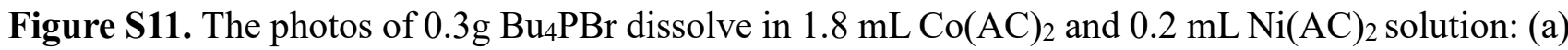
before, (b) after shaking $20 \mathrm{~s}$.

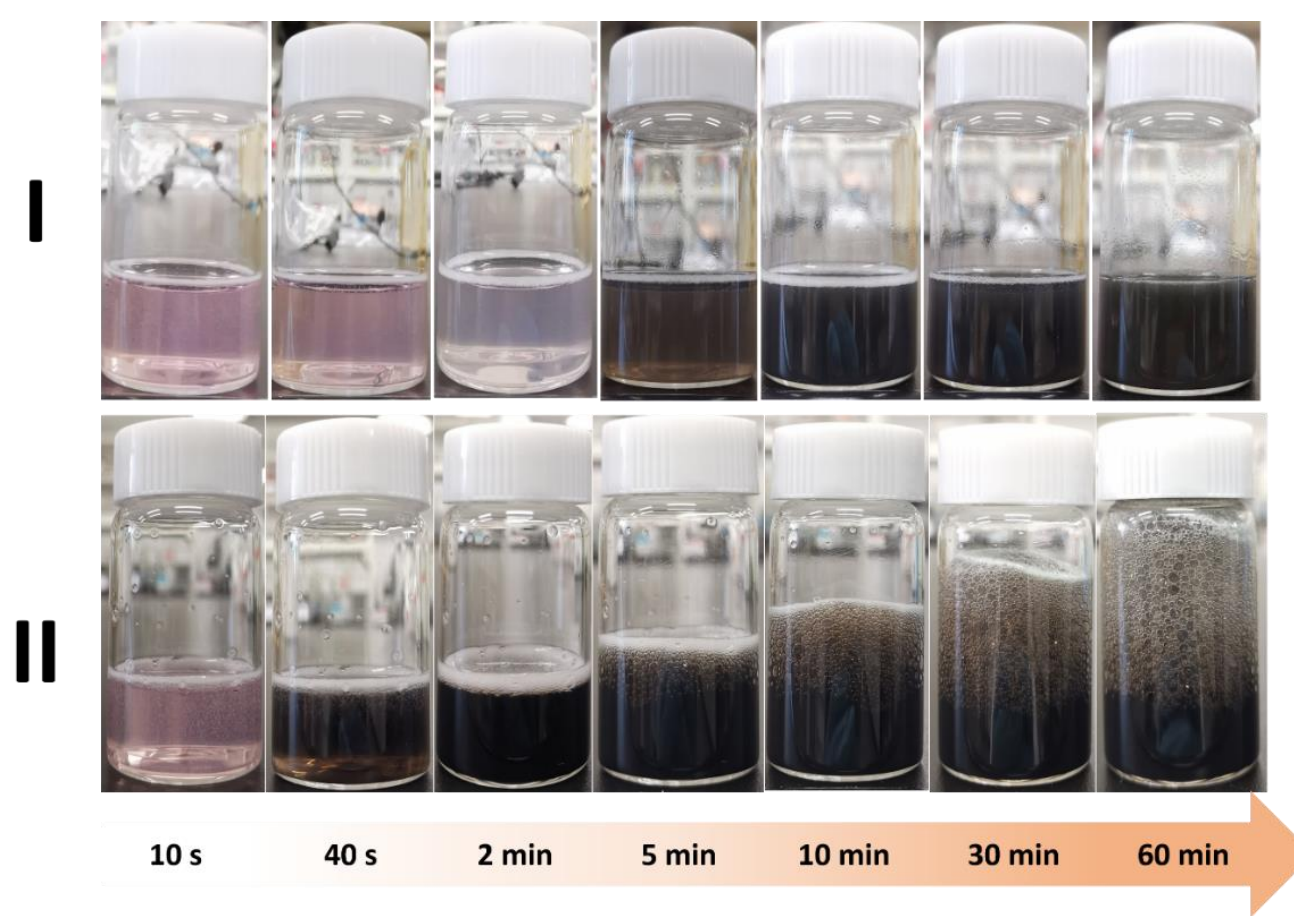

Figure S12. Photos of the reduction process of (I) meso-Ni ${ }_{10.0} \mathrm{Co}_{74.5} \mathrm{~B}_{15.5} \mathrm{AASs}$ with $\mathrm{Bu} 4 \mathrm{PBr}$ and (II) meso- $\mathrm{Ni}_{10.0} \mathrm{Co}_{74.5} \mathrm{~B}_{15.5}$ AASs without $\mathrm{Bu}_{4} \mathrm{PBr}$ (meso-Ni ${ }_{10.0} \mathrm{Co}_{74.5} \mathrm{~B}_{15.5}-2$ ). 

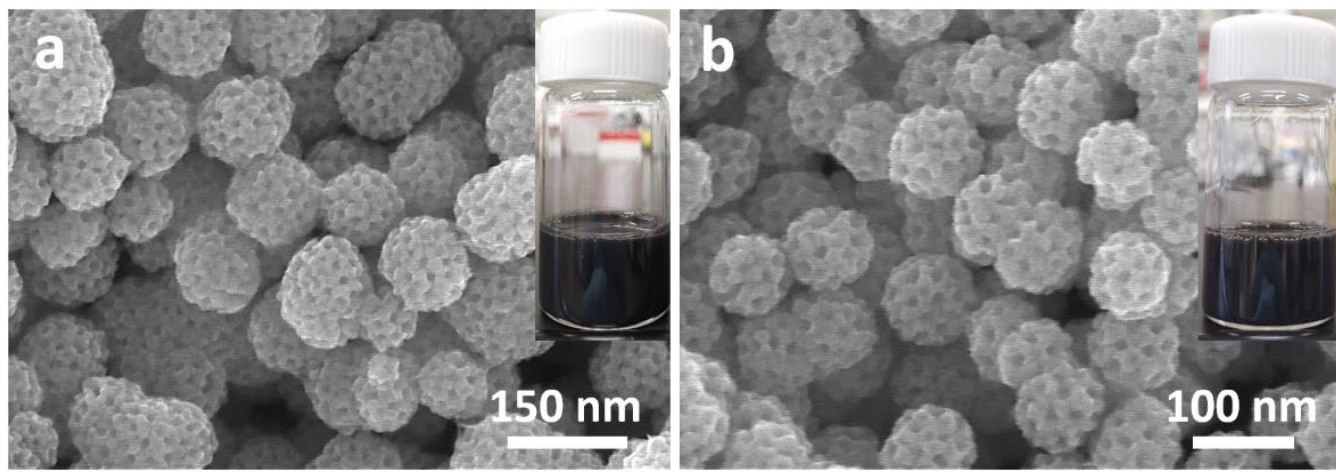

Figure S13. SEM images of meso- $\mathrm{Ni}_{10.0} \mathrm{Co}_{74.5} \mathrm{~B}_{15.5}$ AASs prepared by replacing $\mathrm{Bu} 4 \mathrm{PBr}$ with (a) $\mathrm{Bu}_{4} \mathrm{PCl}$ and (b) $\mathrm{Bu}_{4} \mathrm{NBr}$, respectively. (inset: the corresponding photos after reacting for 1.0 hour)

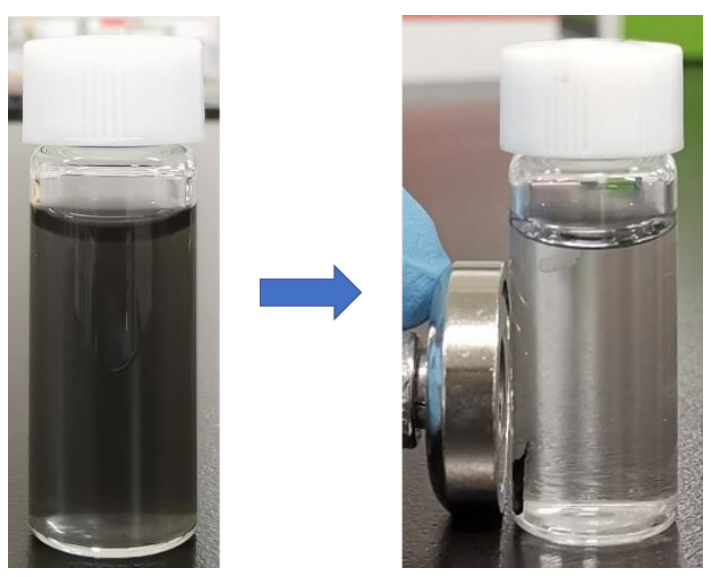

Figure S14. The photographs of the meso- $\mathrm{Ni}_{10.0} \mathrm{Co}_{74.5} \mathrm{~B}_{15.5}$ AASs before (left) and after (right) the magnetic separation. 

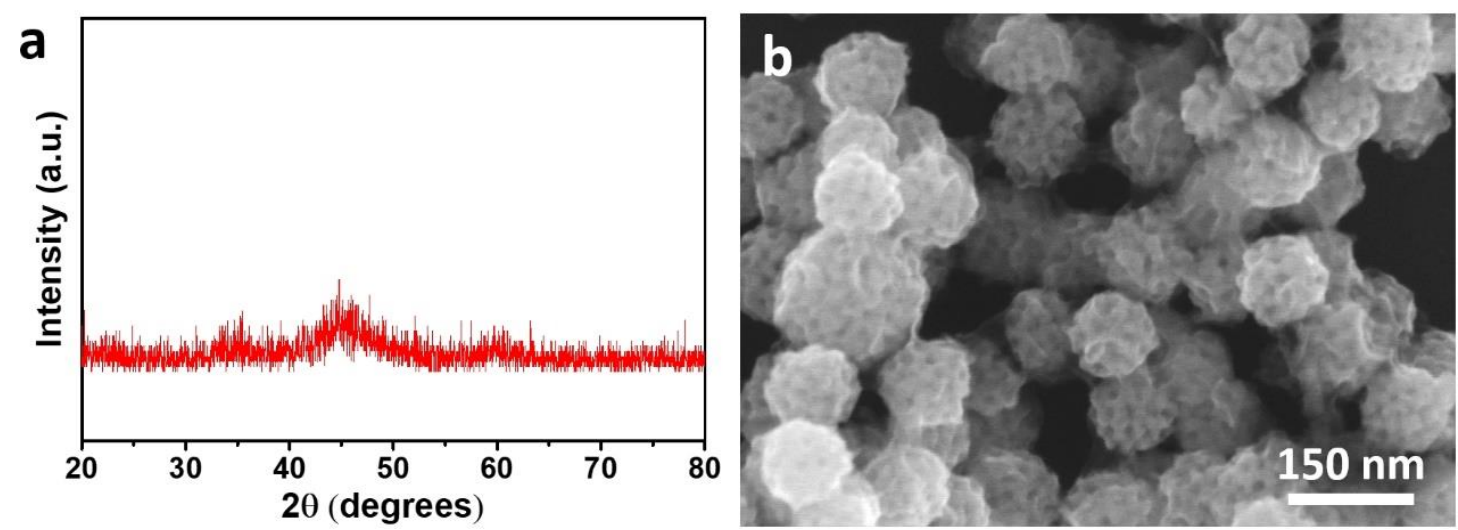

Figure S15. The (a) XRD pattern and (b) SEM image of meso-Ni ${ }_{10.0} \mathrm{Co}_{74.5} \mathrm{~B}_{15.5}$ AAs after the stability test.

Table S1. The relationship between the final elemental concentration and the precursors.

\begin{tabular}{|c|c|c|c|c|c|}
\hline \multirow{3}{*}{ Catalyst } & \multirow{3}{*}{$\begin{array}{l}\text { Feeding ratio of } \\
\qquad \mathrm{Ni}^{2+}: \mathrm{Co}^{2+}\end{array}$} & \multirow{3}{*}{$\begin{array}{c}\text { Final ratio of } \\
\qquad \mathrm{Ni}: \mathrm{Co}\end{array}$} & \multicolumn{3}{|c|}{ Bulk composition in product } \\
\hline & & & \multicolumn{3}{|c|}{$(\%)$} \\
\hline & & & $\mathrm{Ni}$ & Co & B \\
\hline meso-Co ${ }_{85.0} \mathrm{~B}_{15.0}$ & $0: 100$ & $0: 100$ & - & 85.0 & 15.0 \\
\hline meso- & $10: 90$ & $12: 88$ & 10.0 & 74.5 & 15.5 \\
\hline $\mathrm{Ni}_{10.0} \mathrm{Co}_{74.5} \mathrm{~B}_{15.5}$ & & & & & \\
\hline meso- & $20: 80$ & $19: 81$ & 16.3 & 67.8 & 15.9 \\
\hline $\mathrm{Ni}_{16.3} \mathrm{Co}_{67.8} \mathrm{~B}_{15.9}$ & & & & & \\
\hline meso- & $30: 70$ & $29: 71$ & 24.0 & 60.0 & 16.0 \\
\hline $\mathrm{Ni}_{24.0} \mathrm{Co}_{60.0} \mathrm{~B}_{16.0}$ & & & & & \\
\hline meso- & $50: 50$ & $49: 51$ & 40.0 & 41.7 & 18.3 \\
\hline $\mathrm{Ni}_{40.0} \mathrm{Co}_{41.7} \mathrm{~B}_{18.3}$ & & & & & \\
\hline meso- & $80: 20$ & $80: 20$ & 63.1 & 16.1 & 20.8 \\
\hline $\begin{array}{l}\mathrm{Ni}_{63.1} \mathrm{Co}_{16.1} \mathrm{~B}_{20.8} \\
\text { meso-Ni } \mathrm{Ni}_{79.0} \mathrm{~B}_{21.0}\end{array}$ & $100: 0$ & $100: 0$ & 79.0 & - & 21.0 \\
\hline
\end{tabular}


Table S2. Catalytic activities from the hydrolysis of AB catalyzed by as-prepared samples with different metal ratios.

\begin{tabular}{cccc}
\hline Catalyst & $n_{\text {cat. }} / n_{\mathrm{AB}}$ & $\mathrm{T}(\mathrm{K})$ & $\mathrm{TOF}\left(\mathrm{min}^{-1}\right)$ \\
\hline meso-Co $_{85.0} \mathrm{~B}_{15.0}$ & 0.05 & 298 & 4.88 \\
meso-Ni $_{10.0} \mathrm{Co}_{74.5} \mathrm{~B}_{15.5}$ & 0.05 & 298 & 6.50 \\
meso-Ni $_{16.3} \mathrm{Co}_{67.8} \mathrm{~B}_{15.9}$ & 0.05 & 298 & 5.44 \\
meso-Ni ${ }_{24.0} \mathrm{Co}_{60.0} \mathrm{~B}_{16.0}$ & 0.05 & 298 & 4.20 \\
meso-Ni $_{40.0} \mathrm{Co}_{41.7} \mathrm{~B}_{18.3}$ & 0.05 & 298 & 4.13 \\
meso-Ni $_{63.1} \mathrm{Co}_{16.1} \mathrm{~B}_{20.8}$ & 0.05 & 298 & 3.17 \\
meso-Ni $_{79.0} \mathrm{~B}_{21.0}$ & 0.05 & 298 & 2.06 \\
\hline
\end{tabular}

Table S3. The hydrolysis of $\mathrm{AB}$ performance of as-prepared $\mathrm{Ni}_{0.13} \mathrm{Co}_{0.87} \mathrm{~B}$ prepared with different structure-directing agents.

\begin{tabular}{ccccc}
\hline Catalyst & Prepared with & $n_{\text {cat. }} / n_{\mathrm{AB}}$ & $\mathrm{T}(\mathrm{K})$ & $\mathrm{TOF}\left(\mathrm{min}^{-1}\right)$ \\
\hline & PS- $b$ - $\mathrm{PEO}+\mathrm{Bu} 4 \mathrm{PBr}$ & 0.05 & 298 & 6.50 \\
$\mathrm{Ni}_{10.0} \mathrm{Co}_{74.5} \mathrm{~B}_{15.5}$ & $\mathrm{PS}-b-\mathrm{PEO}$ & 0.05 & 298 & 4.73 \\
& $\mathrm{Bu}$ PBr & 0.05 & 298 & 2.92 \\
& None & 0.05 & 298 & 0.66 \\
\hline
\end{tabular}


Table S4. The catalytic activity of different Co-based catalysts used for the hydrolytic dehydrogenation of $\mathrm{AB}$.

\begin{tabular}{|c|c|c|c|c|c|c|}
\hline Catalyst & $\begin{array}{l}\text { Temp. } \\
(\mathrm{K})\end{array}$ & $\mathrm{n}_{\text {cat. }} / \mathrm{n}_{\mathrm{AB}}$ & $\begin{array}{l}\text { Hydrogen generation } \\
\text { rate }\left(\mathrm{mL} \mathrm{min}^{-1} \mathrm{~g}_{\mathrm{cat}}{ }^{-1}\right)\end{array}$ & $\begin{array}{c}\text { TOF } \\
\left(\min ^{-1}\right)\end{array}$ & $\begin{array}{c}E a \\
\left(\mathrm{~kJ} \cdot \mathrm{mol}^{-1}\right)\end{array}$ & Ref. \\
\hline meso-Ni ${ }_{10.0} \mathrm{Co}_{74.5} \mathrm{~B}_{15.5} \mathrm{AASs}$ & 298 & 0.05 & 2469 & 6.50 & 38.62 & This work \\
\hline NiCo-Pt nanopolyhedron & 298 & 0.0146 & - & - & 45.72 & {$[1]$} \\
\hline Co-W-B nanoparticle & 298 & - & 1910 & - & 38 & {$[2]$} \\
\hline Co-Mo-B nanoparticle & 298 & - & 2460 & - & 43 & {$[2]$} \\
\hline Co-B powder & 298 & - & 400 & - & 44 & {$[3]$} \\
\hline $\mathrm{Co}_{0.7} \mathrm{Ni}_{0.3} \mathrm{~B}-1$ & 298 & 0.036 & - & 4.3 & - & {$[4]$} \\
\hline $\mathrm{Co}_{0.7} \mathrm{Ni}_{0.3} \mathrm{~B}-\mathrm{h}$ & 298 & 0.036 & - & 30.9 & 32.3 & {$[4]$} \\
\hline $\mathrm{Co}_{0.75} \mathrm{~B}_{0.25}$ & 333 & - & 7607 & - & 42.10 & {$[5]$} \\
\hline Intrazeolite $\mathrm{Co}(0)$ nanoclusters & 298 & 0.02 & - & - & 56 & {$[6]$} \\
\hline hollow Co-B nanospindles & 293 & 0.013 & 1283 & - & 47.1 & [7] \\
\hline Co-Mo-P-B naoparticle & 298 & - & 2750 & - & 23 & {$[8]$} \\
\hline $\begin{array}{l}\mathrm{Cu}_{0.3} @ \mathrm{Fe}_{0.1} \mathrm{Co}_{0.6} \mathrm{NPs} \text { core- } \\
\text { shell nanoparticles }\end{array}$ & 298 & 0.1 & 6674.2 & 10.5 & 38.78 & [9] \\
\hline $\mathrm{Cu}_{0.2} \mathrm{Co}_{0.8} / \mathrm{HPC}$ & 298 & 0.158 & 2960 & 6.3 & 41.7 & {$[10]$} \\
\hline PLD-deposited Co-B films & 298 & - & 2420 & - & 34 & {$[3]$} \\
\hline $\mathrm{Co}_{0.9} \mathrm{Ni}_{0.1} /$ graphene & 298 & 0.05 & - & 16.4 & 13.49 & {$[11]$} \\
\hline NiCo/MIL-101-1-U & 298 & 0.02 & - & 44.3 & - & {$[12]$} \\
\hline $\mathrm{CoNi} / \mathrm{RGO}$ & 298 & 0.05 & - & 19.54 & 38.89 & {$[13]$} \\
\hline $\mathrm{Co} / \gamma-\mathrm{Al}_{2} \mathrm{O}_{3}$ & 298 & 0.018 & - & 2.27 & 62 & {$[14]$} \\
\hline $\mathrm{Cu}_{0.5} \mathrm{Co}_{0.5} / \mathrm{PDDA}-\mathrm{HNT}$ & $\mathrm{rt}$ & 0.09 & - & 30.8 & 35.15 & {$[15]$} \\
\hline CuCo@MIL-101 & $\mathrm{rt}$ & 0.034 & - & 19.6 & - & [16] \\
\hline
\end{tabular}

Note for Table S4: This table lists some reported supported and unsupported Co-based catalysts for the hydrolysis of ammonia borane. Most of the reported catalysts with higher TOF are based on supported nanoparticles in which the supports such as graphene and MIL can provide numerous contact sites and can anchor the nanoparticles to prevent agglomeration. The purpose of our research is to improve the catalytic performance as high as possible by manufacturing the mesoporous structure and alloy composition to avoid the use of supports. Of course, the activity of unsupported catalysts at present is still not as good as the supported catalysts, which requires further research. 


\section{Reference}

(1) Wen, M.; Zhou, S.; Wu, Q.; Zhang, J.; Wu, Q.; Wang, C.; Sun, Y. Construction of NiCo-Pt Nanopolyhedron Inlay-Structures and Their Highly Efficient Catalysis Hydrolytic Dehydrogenation toward Ammonia Borane. J. Power Sources 2013, 232, 86-92.

(2) Fernandes, R.; Patel, N.; Miotello, A.; Jaiswal, R.; Kothari, D. C. Dehydrogenation of Ammonia Borane with Transition Metal-Doped Co-B Alloy Catalysts. Int. J. Hydrogen Energ. 2012, 37, 2397-2406.

(3) Patel, N.; Fernandes, R.; Guella, G.; Miotello, A. Nanoparticle-Assembled Co-B Thin Film for The Hydrolysis of Ammonia Borane: A Highly Active Catalyst for Hydrogen Production. Appl. Catal. B: Environ. 2010, 95, 137-143.

(4) Wang, H.; Gao, D.; Wang, L.; Chi, Y.; Wang, M.; Gu, Y.; Wang, C.; Zhao, Z. Highly Dispersed Surfactant-Free Amorphous NiCoB Nanoparticles and Their Remarkable Catalytic Activity for Hydrogen Generation from Ammonia Borane Dehydrogenation. Catal. Letters 2018, 148, 17391749.

(5) Kantürk Figen, A. Dehydrogenation Characteristics of Ammonia Borane via Boron-Based Catalysts (Co-B, Ni-B, Cu-B) under Different Hydrolysis Conditions. Int. J. Hydrogen Energ. 2013, 38, 9186-9197.

(6) Rakap, M.; Özkar, S. Hydrogen Generation from The Hydrolysis of Ammonia-Borane Using Intrazeolite Cobalt(0) Nanoclusters Catalyst. Int. J. Hydrogen Energ. 2010, 35, 3341-3346.

(7) Tong, D. G.; Zeng, X. L.; Chu, W.; Wang, D.; Wu, P. Magnetically Recyclable Hollow Co-B Nanospindles as Catalysts for Hydrogen Generation from Ammonia Borane. J. Mater. Sci. 2010, $45,2862-2867$.

(8) Fernandes, R.; Patel, N.; Paris, A.; Calliari, L.; Miotello, A. Improved $\mathrm{H}_{2}$ Production Rate by Hydrolysis of Ammonia Borane Using Quaternary Alloy Catalysts. Int. J. Hydrogen Energ. 2013, 38, 3313-3322.

(9) Qiu, F.; Dai, Y.; Li, L.; Xu, C.; Huang, Y.; Chen, C.; Wang, Y.; Jiao, L.; Yuan, H. Synthesis of $\mathrm{Cu} @$ FeCo Core-Shell Nanoparticles for The Catalytic Hydrolysis of Ammonia Borane. Int. J. Hydrogen Energ. 2014, 39, 436-441.

(10)Wang, H.; Zhou, L.; Han, M.; Tao, Z.; Cheng, F.; Chen, J. CuCo Nanoparticles Supported on Hierarchically Porous Carbon as Catalysts for Hydrolysis of Ammonia Borane. J. Alloy Compd. 
2015, 652, 382-388.

(11)Feng, W.; Yang, L.; Cao, N.; Du, C.; Dai, H.; Luo, W.; Cheng, G. In Situ Facile Synthesis of Bimetallic CoNi Catalyst Supported on Graphene for Hydrolytic Dehydrogenation of Amine Borane. Int. J. Hydrogen Energ. 2014, 39, 3371-3380.

(12)Liu, P.; Gu, X.; Kang, K.; Zhang, H.; Cheng, J.; Su, H. Highly Efficient Catalytic Hydrogen Evolution from Ammonia Borane Using The Synergistic Effect of Crystallinity and Size of NobleMetal-Free Nanoparticles Supported by Porous Metal-Organic Frameworks. ACS Appl. Mater. Interfaces 2017, 9, 10759-10767.

(13) Yang, Y.; Zhang, F.; Wang, H.; Yao, Q.; Chen, X.; Lu, Z.-H. Catalytic Hydrolysis of Ammonia Borane by Cobalt Nickel Nanoparticles Supported on Reduced Graphene Oxide for Hydrogen Generation. J. Nanomater. 2014, 2014, 294350.

(14)Xu, Q.; Chandra, M. Catalytic Activities of Non-Noble Metals for Hydrogen Generation from Aqueous Ammonia-Borane at Room Temperature. J. Power Sources 2006, 163, 364-370.

(15)Liu, Y.; Zhang, J.; Guan, H.; Zhao, Y.; Yang, J.-H.; Zhang, B. Preparation of Bimetallic Cu-Co Nanocatalysts on Poly (Diallyldimethylammonium Chloride) Functionalized Halloysite Nanotubes for Hydrolytic Dehydrogenation of Ammonia Borane. Appl. Surf. Sci. 2018, 427, 106113.

(16)Li, J.; Zhu, Q.-L.; Xu, Q. Non-Noble Bimetallic CuCo Nanoparticles Encapsulated in The Pores of Metal-Organic Frameworks: Synergetic Catalysis in the Hydrolysis of Ammonia Borane for Hydrogen Generation. Catal. Sci. Technol., 2015, 5, 525-530. 